\title{
Monitoring oxidation changes in commercial extra virgin olive oils with fluorescence spectroscopy-based prototype
}

\author{
Puneet Mishra · Lourdes Lleó - Teresa Cuadrado - Margarita Ruiz-Altisent • \\ Natalia Hernández-Sánchez
}

\begin{abstract}
Maintaining the quality of extra virgin olive oils (EVOOs) in the current globalised market is a key challenge for the olive oil manufacturers. To maintain the quality, one major factor is to resist its oxidation during processing and storage. Resisting the oxidation in EVOOs is a challenging task requiring control of different physical and chemical factors. However, rapid, real-time, and non-destructive monitoring the oxidation in EVOOs could support in maintaining its quality during storage. The present study utilises a novel right-angle fluorescence spectroscopy-based prototype for rapid monitoring of oxidation changes in three different EVOOs varieties (Arbequina, Picual, and Cornicabra) during storage. Furthermore, the effects of light exposure on oxidation of EVOOs were also studied. Two major changes in the fluorescence spectra of EVOO samples were mainly observed: a decrease in fluorescence emission intensity at $671 \mathrm{~nm}$, and an increase in fluorescence intensities at 420 , 440,464 , and $515 \mathrm{~nm}$. These two changes were related to a decrease in chlorophyll content and parallel formation of oxidation products. The formation of oxidation products was found to have a linear relationship with the decrease in chlorophyll content. Out of the three, the Cornicabra variety showed the highest rate of oxidation changes. Furthermore, the EVOOs degraded rapidly when get exposed to light. In a fast and non-destructive manner, the fluorescence spectroscopy-based prototype successfully monitored the oxidation changes in the EVOOs.
\end{abstract}

Keywords Extra virgin olive oil - Oxidation - Storage Light exposure

$\begin{array}{ll}\text { Abbreviations } \\ \left({ }^{1} \mathrm{O}_{2}\right) & \text { Singlet oxygen } \\ \left({ }^{3} \mathrm{O}_{2}\right) & \text { Triplet oxygen } \\ \text { EU } & \text { European Union } \\ \text { EVOOs } & \text { Extra virgin olive oils } \\ \text { nm } & \text { Nanometre } \\ \text { UV-Vis } & \text { Ultraviolet and visible } \\ \text { VOOs } & \text { Virgin olive oils }\end{array}$

\section{Introduction}

Extra virgin olive oils (EVOOs) being an important source of antioxidants and healthy fats is an economically important product of Mediterranean countries [1]. EVOOs are obtained from the fruit of the olive tree by minimal processing maintaining its natural flavour and health beneficial characteristics [2]. Furthermore, EVOOs are the most recommended choice for the consumer among the different grades of olive oils [3]. However, the production of olive oil is limited to a certain period of time during the year; therefore, to maintain its commercial availability, packaging materials such as glass, plastic, and metal are used [4]. During storage, due to the characteristic property of different storage materials such as transparency, the EVOOs get exposed to the natural light conditions promoting the photo-oxidation of oils [5]. In addition, even under dark conditions, the oils deteriorate due to auto-oxidation [6]. The oxidation of EVOOs leads to the formation of primary oxidation products such as hydroperoxides, which further decompose to secondary oxidation products like aldehydes, alcohols, and ketones [6, 7]. These latter formed compounds are responsible for the 
characteristic off-flavour in oils, leading to an overall quality degradation.

Due to the existing globalised market of EVOOs, the producer needs to maintain a high quality of their products $[8,9]$. European Union (EU) has set up regulations which include specific ranges of different quality parameters for different grades of commercial olive oils. One such regulated designation for EVOOs and Virgin Olive Oils (VOOs) (EEC No. 2568/91 and subsequent amendments) includes a high resistance to oxidative deterioration due to the triacylglycerol composition with the high content of monounsaturated fatty acids, and to a group of phenolic antioxidants, especially polyphenols and tocopherols. To maintain the high quality of EVOOs, control of external factors, i.e., oxygen concentration, temperature, light, darkness, and ultraviolet blockers, or colour of containers $[10,11]$, and internal factors, i.e., major and minor chemical constituents of EVOO samples that influence oxidation [12-14] is usually performed. Often different techniques such as gas chromatography-mass spectroscopy and high-performance liquid chromatography are used to monitor, characterize, and discriminate the quality and origin of the olive oils. These techniques are highly sensitive and many recent applications can also be found [15-20]. However, the main disadvantage of these highly advanced techniques is the cost, complexity, and need of skilled manpower to run the experiments.

The previous works performed with fluorescence spectroscopy proved it a promising tool to deal with authentication and quality monitoring of olive oils [21-23]. One major advantage of fluorescence spectroscopy over other conventional methods is its minimum sample preparation requirement with the absence of solvents and reagents. In more to that, applications of analytical methods based on fluorescence to olive oils have a high potential, because many interesting minor components related to the status of oxidation of olive oils are fluorescent molecules and exhibit characteristic signatures at different wavelengths such as chlorophylls and pheophytins $(600-750 \mathrm{~nm})[24,25]$, antioxidants compounds such as polyphenols and $\alpha$-tocopherol (300-390 nm) $[26,27]$, compounds derived from vita$\min \mathrm{E}(\sim 525 \mathrm{~nm})$ and hydrolysis products $(455 \mathrm{~nm})$ [24], and primary and secondary oxidation products $(410-450$, $450-480$, and $485-540 \mathrm{~nm})[24,28]$. Therefore, monitoring the evolution of the fluorescence emission spectra can support understanding the changes in the contents of such minor compounds.

In a similar application to our work, Kalua et al. [29] stored VOO samples in light, dark, and low-temperature dark conditions for 12 months, and monitored evolution with fluorescence spectroscopy. The results showed that the oil samples stored at low temperature and in darkness maintained characteristics closest to the fresh oil samples, while oil stored in the light showed the largest deviation from freshness. Similarly, Sikorska et al. [11] used fluorescence spectroscopy to monitor the quality of olive oils while storage. In their study, olive oils were stored in clear and green glass bottles exposed to light, and in darkness. With storage time and different storage conditions, the results showed that certain compounds like chlorophyll, polyphenol get decreased, and some new unidentified oxidation compounds arose. The changes in the fluorescence spectrum of the oils allowed them to monitor the change in the quality of virgin olive oil. Cheikhousman et al. [30] monitored polyphenols and vitamin E evolution and hydroperoxides degradation with fluorescence spectroscopy. The emission at $330 \mathrm{~nm}$ was considered to assess the phenols and vitamin E contents, and the emission at $450 \mathrm{~nm}$ associated with the newly formed molecules arising from the oxidation processes. Similarly, Tena et al. [31] studied the thermal degradation of VoOs and found that the emission in the ranges of $390-630 \mathrm{~nm}$ allowed the explanation of the increase of the percentage of polar compounds during the experiment.

The aim of the present study was to monitor the oxidation changes in the commercial EVOOs utilising a novel rightangle fluorescence spectroscopy-based prototype. The work explores the fluorescence characteristics of three different commercial EVOOs (Arbequina, Picual, and Cornicabra) and identifies different wavebands regions explaining the effects of oxidation. The focus of the study was on both photo-oxidation and auto-oxidation of the EVOOs and therefore, the work also studies the effect of light exposure on the fluorescence characteristics of EVOOs.

\section{Material and methods}

\section{Samples}

The work was performed in two parts, the first part was devoted to capturing the effects of the storage under natural light conditions on the fluorescence characteristics of three different commercial EVOO samples, and the second part included monitoring the fluorescence spectra of EVOO samples during storage in two light conditions: (a) dark and (b) exposed to light conditions.

For the first experiment, three commercial EVOO samples belonging to three different cultivars Arbequina, Picual, and Cornicabra were monitored. The three EVOOs were provided by the Casa de Hualdo manufacturer based in Camino de la barca s/n 45533, El Carpio de Tajo, Toledo, Spain. Samples were divided into aliquots and stored in transparent glass bottles under indirect sunlight conditions, simulating market or house shelves storage conditions. During each experimental date ( 23 dates of measurements during 4 months, from April 2015 to July 2015), fluorescence spectra were acquired from the 
corresponding aliquot of each cultivar leading to a total of 69 spectra resulting from 3 samples $\times 23$ dates.

For the second experiment, additional two samples of Picual were considered. The two samples were divided into aliquots and stored under two different conditions, dark and light conditions, for 6 months (from June 2015 to December 2015). Acquisition of spectra was performed monthly leading to a total number of 24 spectra resulting from 2 samples $\times 2$ conditions $\times 6$ dates. Further details of the EVOO samples are presented in Table 1.

The principle of right-angle prototype setup is illustrated in Fig. 1. The prototype was called right angle because of the incoming direction of the wave of excitation and the outgoing wave of emission from $90^{\circ}$ with the faces of the cuvette containing the sample oil. The assembly of the prototype has two optical filters. The optical filter coupled with fibre coming from light source $S$ allows the passage of beams at wavelengths below $400 \mathrm{~nm}$. The other optical filter coupled to fibre $R$ limits the emitted beams to wavelengths higher than $400 \mathrm{~nm}$. The prototype allows the fast evaluation of the samples based on the registration of the signal resulting from a simultaneous excitation with a wide range of wavelengths (200-400 nm). Different parts of the system were designed, such that the incident and receiving beams were centred within the width of the face of the quartz cuvette, and have the same height relative to the base of the cuvette.

The acquisition with the prototype can be carried out using quartz cuvettes for sample presentation $(10 \mathrm{~mm} \times 10 \mathrm{~mm} \times 45 \mathrm{~mm})$. For a typical experiment, the light from the lamp passes through a filter $(<400 \mathrm{~nm})$ and interacts with the oil sample placed in the cuvette inside of the dedicated opening in prototype for sample presentation. This light is absorbed by the fluorescent compounds; these compounds attain a higher energy state, and while returning to the ground energy state emits fluorescence. This fluorescence light has the characteristic of presenting lower energy than excitation light and, therefore, has longer wavelengths than the excitation. Fluorescence emission passes through a second filter $(>400 \mathrm{~nm})$ and detected by the spectrophotometer, which is further visualized and stored in the attached computer system.

\section{Spectral acquisition}

The spectral measurements were performed with a Hamamatsu photonic multi-channel spectrophotometer connected to the right-angle prototype. The spectrophotometer has 1024 linear silicon detectors with corresponding diffraction grating ranging $196-958 \mathrm{~nm}$. A UV-Vis deuterium lamp
Table 1 Summary of the study and characteristics of the extra virgin olive oil samples obtained at retail markets
Fig. 1 Illustration of rightangle prototype setup for acquiring fluorescence emission spectra of EVOO samples. The part indicated as $S$ is connected to the light source, and at exactly $90^{\circ}$, the part indicated as $R$ is connected to the spectrophotometer for collecting the emission spectrum. The prototype has a central space to place the sample in the cuvette and perform the acquisition

\begin{tabular}{|c|c|c|c|c|c|}
\hline Experiments & Sample (cultivar) & & Light conditions & $\begin{array}{l}\text { Container at } \\
\text { retail market }\end{array}$ & Best before date \\
\hline Storage time & $\begin{array}{l}\text { A (Arbequina) } \\
\text { P1 (Picual) } \\
\text { C (Cornicabra) }\end{array}$ & & Indirect sunlight & Dark glass & $12 / 2015$ \\
\hline Storage condition & $\begin{array}{l}\text { P2 (Picual) } \\
\text { P3 (Picual) }\end{array}$ & $\begin{array}{l}\text { P2_L } \\
\text { P2_D } \\
\text { P3_L } \\
\text { P3_D }\end{array}$ & $\begin{array}{l}\text { Indirect sunlight } \\
\text { Darkness } \\
\text { Indirect sunlight } \\
\text { Darkness }\end{array}$ & Dark glass & $12 / 2015$ \\
\hline
\end{tabular}

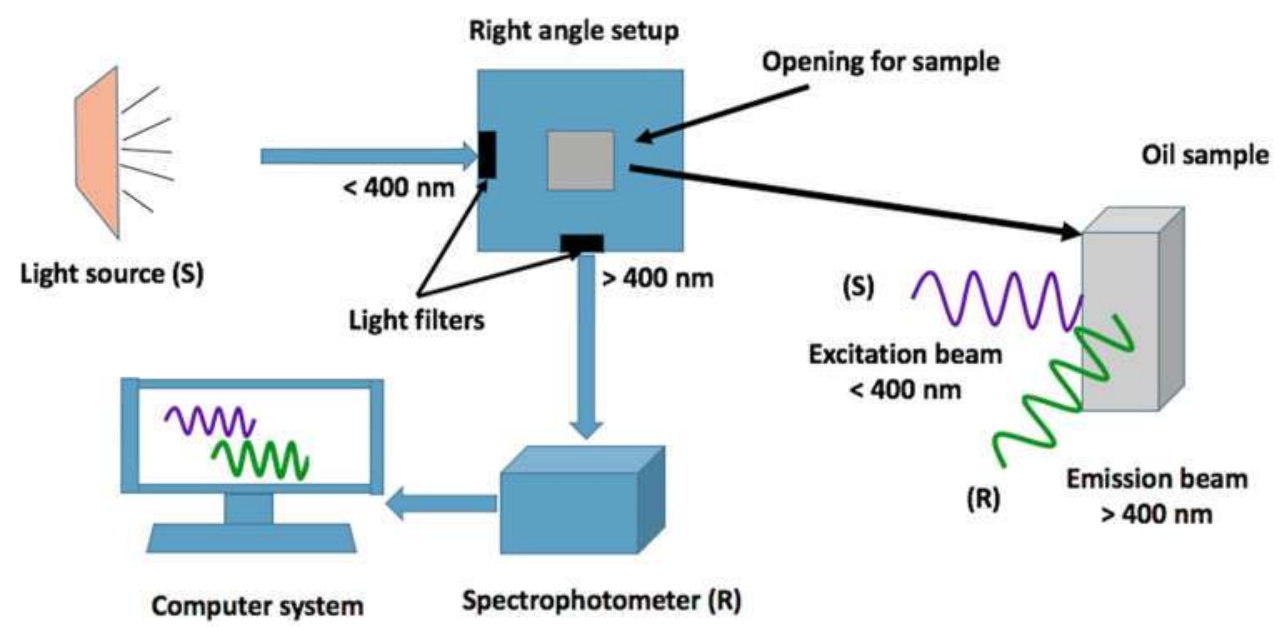


was used as the lightening source to simultaneously excite the fluorophores present in the oils within a range of wavelengths from 200 to $400 \mathrm{~nm}$. Every experimentation day, the samples were taken from the corresponding bottles and filled in cuvettes for sample presentation to the prototype.

\section{Statistical processing}

\section{Data pre-treatment}

Pre-processing of the fluorescence spectra is an important step to remove the non-chemical biases and effects raised due to random noises. The used spectrophotometer has a spectral range of 196-958 $\mathrm{nm}$ for recording the signals. Therefore, all the recorded fluorescence emission spectra have intensity values for complete wavelength range. In the present case, an optical filter to limit the emission wavelengths (>400 nm) was used; therefore, for the wavelength less than $400 \mathrm{~nm}$, the information contained in spectra was not taken into account for our analysis. In addition, the spectral range above $800 \mathrm{~nm}$ was noisy. Before any further analysis, it was decided to reduce the spectral range to $400-800 \mathrm{~nm}$ for all the acquired spectra. To reduce the random noise in the selected spectral range, Savitzky-Golay [32] smoothing was performed. The smoothing was performed with a 15 -point window and polynomials of second order. The polynomials were fitted to the number of points surrounding it, and an estimate of the value of the function was calculated from the fit resulting in the smooth functions applied to the spectra.

Further each spectrum was normalised independently by dividing by the sum of its emission intensities for complete wavelength range and further expressed as its percentage. The normalisation can be understood as follows:

$S_{n}=\frac{S}{\sum_{i=1}^{n} S_{i}} \times 100$,

where $S$ and $S_{n}$ represent the emission spectrum for complete wavelength, raw and normalised, respectively, and $S_{i}$ corresponds to the emission intensity at a particular wavelength. For future processing, pre-processed spectra were used.

\section{Data processing}

Fluorescence spectra acquired at each experimental date from a sample were arranged along the rows of a matrix of fluorescence intensities. Since fluorescence intensity at specific wavelengths can be related to the presence of their corresponding fluorophores; therefore, as a first step, it was decided to identify the different peaks which were evolving during the experimentation period. The standard deviation for each wavelength was calculated for the complete period of observation to identify the peaks that evolved more intensely in comparison to their surrounding regions over the fluorescence spectra.

Once the peaks were identified, the evolution of their local maxims was monitored. This was performed by subtracting the first day fluorescence spectra from all the spectra obtained in following experimental dates for each oil independently. The subtraction revealed the quantitative changes occurred in the oil from the first day of observation. Furthermore, linear curve fitting was performed to estimate the rate of evolution for each EVOO sample. The linear fitting was performed with help of bisquare robustness method. This method increases the robustness of the linear fit by minimising a weighted sum of squares, where the weight given to each data point depends on how far the point is from the fitted line. Points near the line get full weight. Points farther from the line get reduced weight. For most cases, the bisquare weight method simultaneously seeks to find a curve that fits the bulk of the data using the usual leastsquares approach, and it minimises the effect of outliers. From the obtained parameters of linear fits, the slope values were used to explain the rate of evolution of different fluorescence regions in different EVOO samples.

All data analysis was performed using MATLAB_R2014a version 8.3.0.532 (The Mathworks, Natwick, USA) software. The curve fitting was performed with the MATLAB's inbuilt "Curve Fitting" application.

\section{Results and discussion}

\section{Spectra}

The fluorescence spectra of different EVOO samples for 23 days of monitoring before and after pre-processing are presented in Fig. 2. Figure $2 a-c$ shows the raw fluorescence spectra for Arbequina (A), Picual (P1), and Cornicabra (C), respectively. In raw spectra, a relatively high fluorescence intensity peak around $671 \mathrm{~nm}$ and a shoulder peaks at $735 \mathrm{~nm}$ can be seen for all EVOO samples. These peaks were related with the fluorescence of chlorophylls and pheophytins [24, 25], usually present in EVOO samples and responsible for imparting the green colour to the oil. Typically, the peak around $660-700 \mathrm{~nm}$ originates from the emission of pigments related to chlorophyll group, chlorophyll $a$ and $b$, and pheophytins a and $b$ [33].

As revealed in Fig. 2, the peaks at $671 \mathrm{~nm}$ and shoulder around $735 \mathrm{~nm}$ evolved to lower intensity values as storage time increased. To depict the evolution of spectra with storage time, the degrading colours were assigned to spectra, where spectra in darkest red represent the first observation and the lightest red represents the last one. It can also be noted in Fig. 2a-c that the high-intensity peaks at $671 \mathrm{~nm}$ and at $735 \mathrm{~nm}$ overlaid the visualization of other smaller 


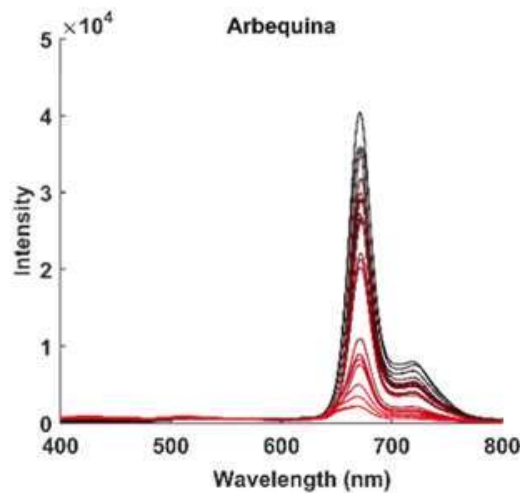

(a)

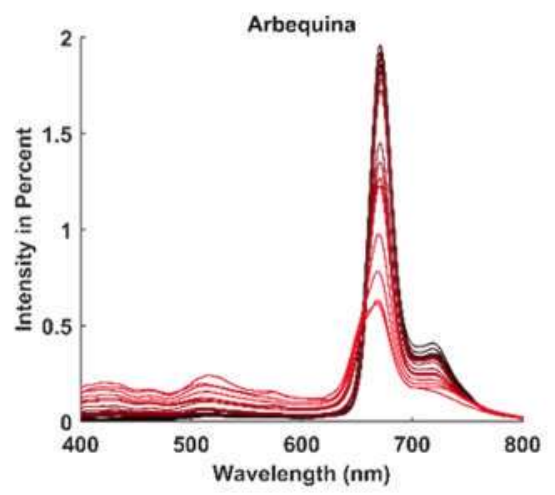

(d)

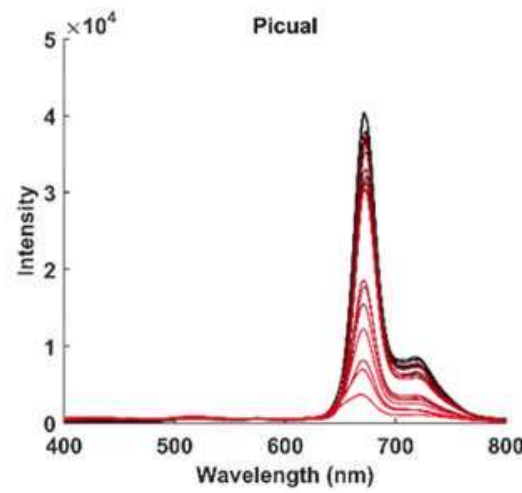

(b)

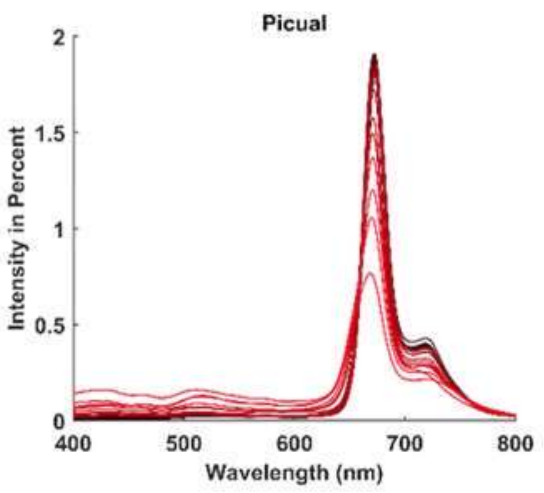

(e)

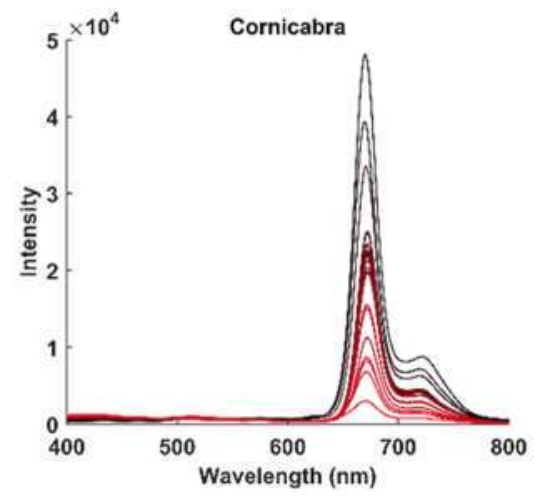

(c)

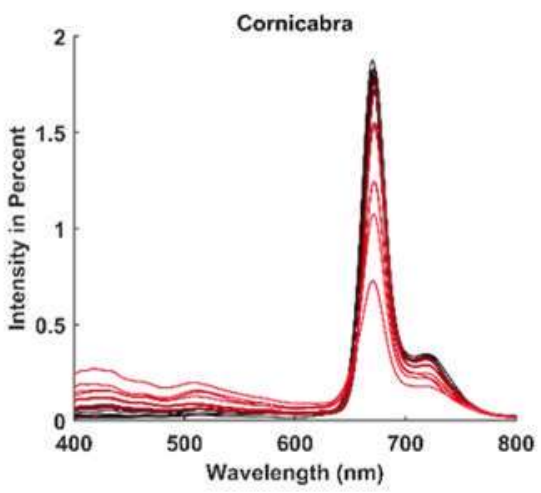

(f)
Fig. 2 Fluorescence spectra obtained for three different EVOO samples, 23 days of monitoring during 4 months. Before pre-processing: a Arbequina, b Picual (P1), and c Cornicabra; after pre-processing:

peaks resulting from different underlying chemical constituents such as vitamin $\mathrm{E}$ ( $~ 525 \mathrm{~nm}$ ), hydrolysis products $(455 \mathrm{~nm})$ [24], and primary and secondary oxidation products $(410-450,450-480$, and $485-540 \mathrm{~nm})[24,28]$.

Figure $2 \mathrm{~d}-\mathrm{f}$ shows the pre-processed fluorescence spectra for Arbequina (A), Picual (P1), and Cornicabra (C), respectively. Spectral pre-processing revealed various peaks in the region of 400-600 nm, whose fluorescence intensity increased with the simultaneous decrease in the intensity at $671 \mathrm{~nm}$. These observations were in agreement with a previous study performed by Sikorska et al. [11], regarding monitoring of EVOO samples during storage with total luminescence and synchronous scanning fluorescence spectroscopy, and the study by Hernandez-Sanchez et al. [23]. With storage time and different storage conditions, Sikorska et al. [11] and Hernandez-Sanchez et al. [23] found that certain compounds like chlorophyll $(670 \mathrm{~nm})$ and polyphenol decreased and some new oxidation compounds aroused in the intermediate range of $405-670 \mathrm{~nm}$.

The region between 400 and $600 \mathrm{~nm}$ is typically the region for fluorescence peaks related to primary and secondary oxidation products $(410-450,450-480$, and d Arbequina, e Picual (P1), and f Cornicabra. The gradient of colour from darkest red (first day of monitoring) to lightest red (last day of monitoring) is used

$485-540 \mathrm{~nm})[24,28]$. These primary and secondary oxidation products often result from the auto-oxidation and photo-oxidation of oils occurring during the storage, in which triplet oxygen $\left({ }^{3} \mathrm{O}_{2}\right)$ and singlet oxygen $\left({ }^{1} \mathrm{O}_{2}\right)$ react with the oil, respectively [34]. The oxidation of oil can get influenced by various factors such as the pigments, peroxides, antioxidants, fatty acid composition of the oil, oil refining, external energy (heat or light), and the concentration of oxygen. However, differentiating the effects arising due to these various factors is not an easy task. The primary oxidation products are relatively stable at room temperature; however, in high temperature, they decompose to alkoxy radicals and then form aldehydes, ketones, acids, esters, alcohols, and short-chain hydrocarbons. Furthermore, the primary oxidation products lead to the formation of secondary oxidation products. Particularly, in olive oils, the secondary oxidation products are formed immediately after hydroperoxides formation, and the resulting decomposition products of hydroperoxides cause off-flavour in the oxidised oils [34].

The major reason for the change in spectra properties of EVOOs placed in exposed light conditions can be 
Fig. 3 Standard deviation for the fluorescence spectra of three different EVOO samples (blue for Arbequina, red for Picual, and yellow for Cornicabra). The vertical lines in red identify the wavelengths showing local maxima
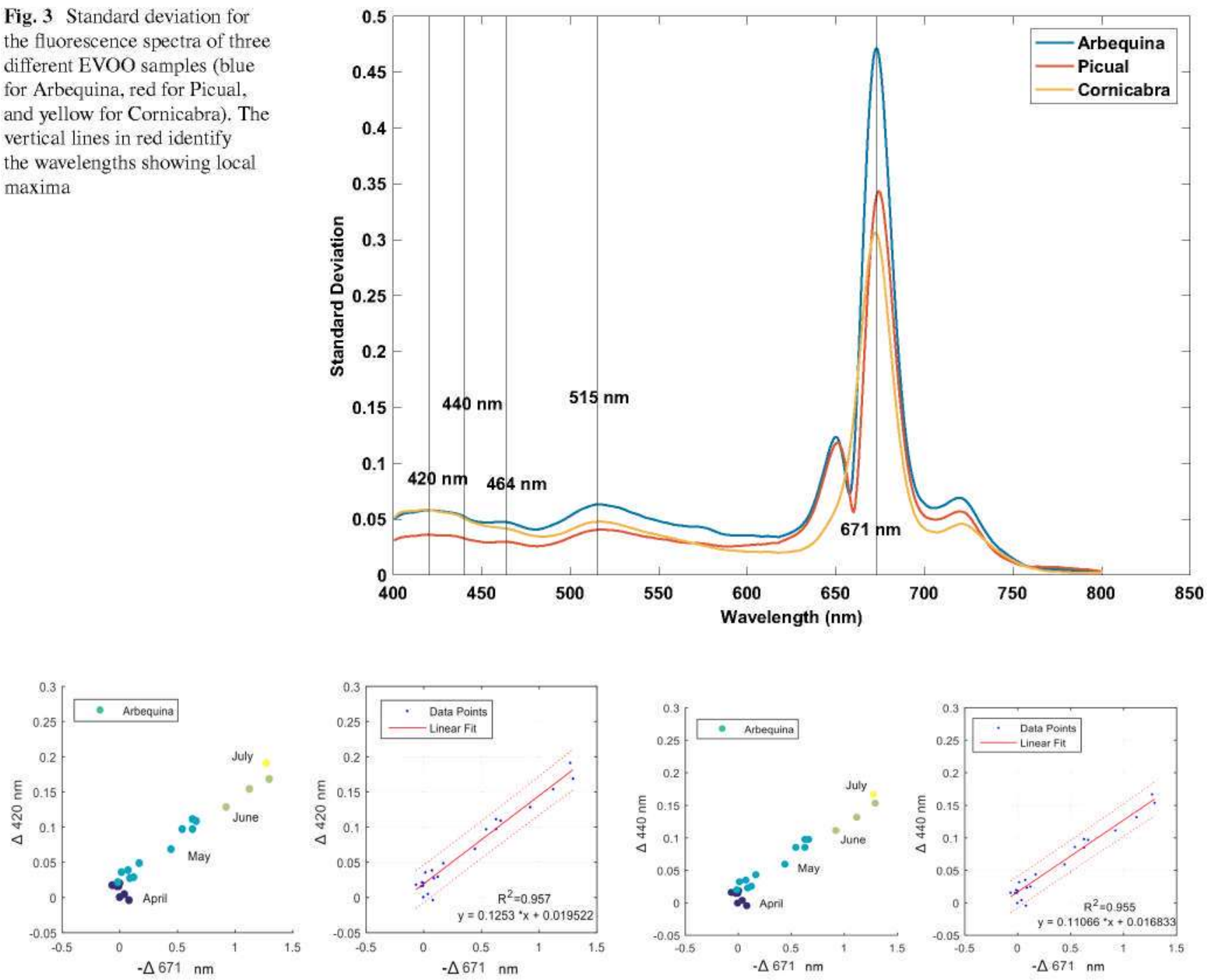

(a)
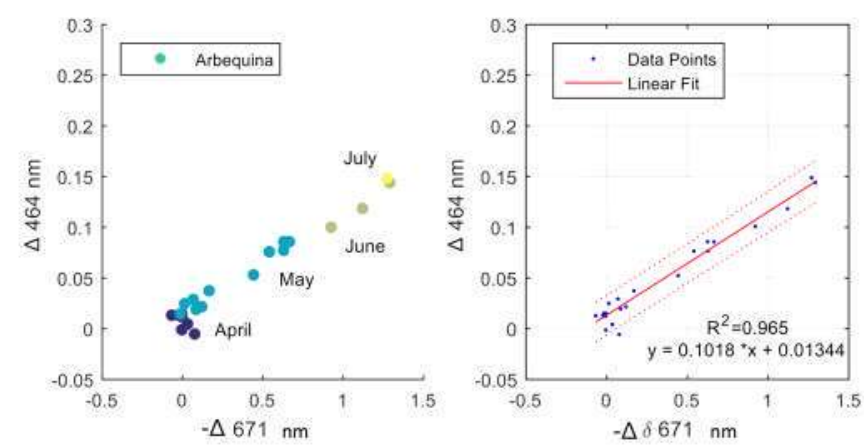

(c)

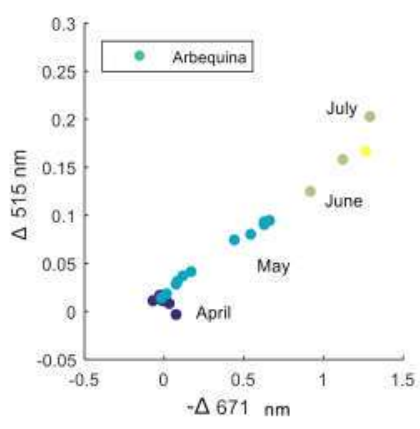

(b)

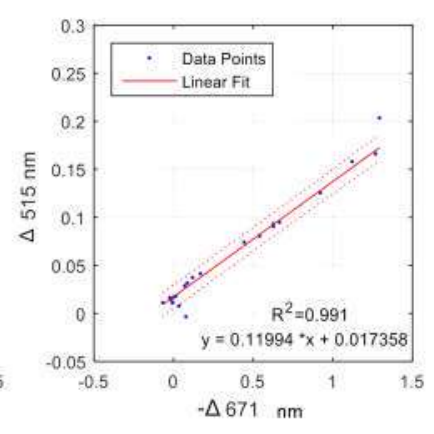

(d)

$515 \mathrm{~nm}$ (d) (y-axis). The oils were monitored for 4 months April, May, June, and July, which are marked in dark blue, sky blue, green, and yellow, respectively

and secondary oxidation products are inter-related processes. Typically, the EVOO samples contain a considerable amount of chlorophyll to promote the photo-oxidation. is a photosensitizer and, under light exposure, acts as prooxidant. Thus, its degradation and the appearance of primary
Fig. 4 Arbequina oil evolution with storage time and the fitted linear curve with $95 \%$ confidence. The evolution is presented individually for $671 \mathrm{~nm}$ (x-axis) vs $420 \mathrm{~nm}$ (a), $440 \mathrm{~nm}$ (b), $464 \mathrm{~nm}$ (c), and

understood as the accelerated oxidation. The chlorophyll 


\section{Peaks selection}

Figure 3 shows the standard deviation plot obtained from the fluorescence spectra of three different EVOO samples (blue for Arbequina, red for Picual, and green for Cornicabra). Five local maxims were identified at $671,515,464,440$, and $420 \mathrm{~nm}$. Similar peaks have been also noticed in the different works corresponding to chlorophylls and pheophytins $(600-750 \mathrm{~nm})[24,25]$, vitamin $\mathrm{E}(\sim 525 \mathrm{~nm})$, hydrolysis products $(455 \mathrm{~nm})$ [24], and primary and secondary oxidation products $(410-450,450-480$, and $485-540 \mathrm{~nm})[24$, 28].

\section{Monitoring evolution}

Figures 4, 5, 6 shows the evolution of the wavelengths showing local maxima, as identified from the standard deviation plot presented in Fig. 3, for Arbequina, Picual, and Cornicabra, respectively. The evolutions are presented for 4 month monitoring period and are identified with different coloured markers as dark blue, sky blue, green, and yellow, for April, May, June, and July, respectively. From the peaks identified in Fig. 3, it can be seen that the peaks at 420, 440, 464 , and $515 \mathrm{~nm}$ are increasing in intensity with storage time. However, the peak at $671 \mathrm{~nm}$ related to chlorophyll
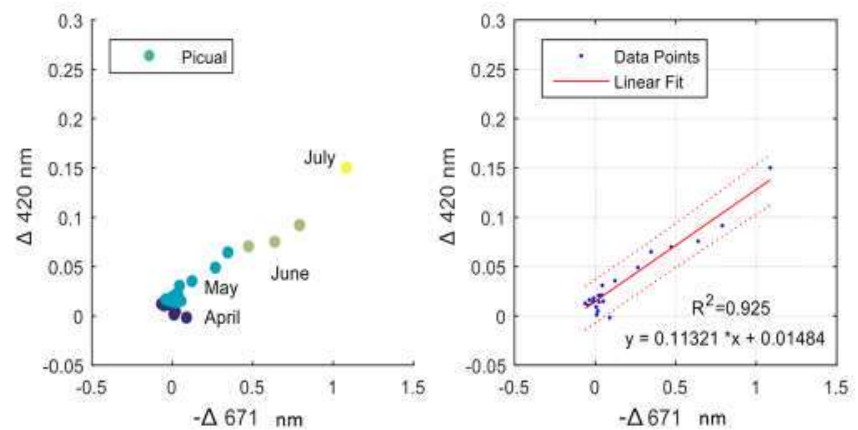

(a)
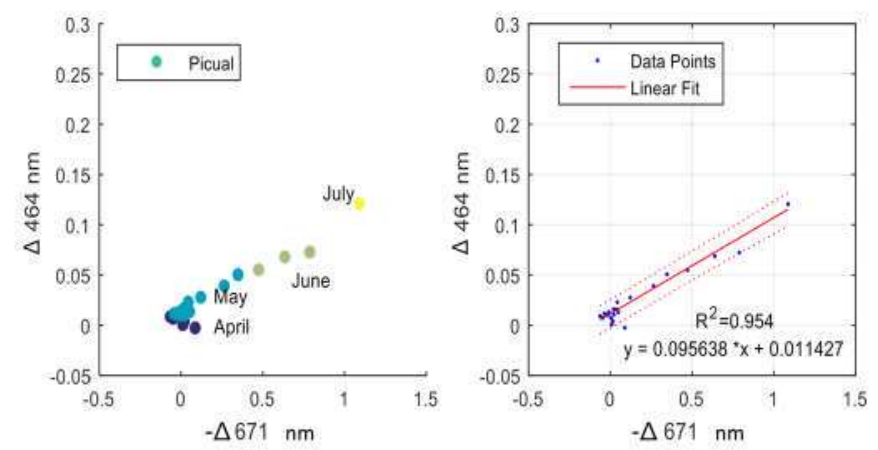

(c)

Fig. 5 Picual oil evolution with storage time and the fitted linear curve with $95 \%$ confidence. The evolution is presented individually for $671 \mathrm{~nm}$ ( $x$-axis) vs $420 \mathrm{~nm}$ (a), $440 \mathrm{~nm}$ (b), $464 \mathrm{~nm}$ (c), and is decreasing in intensity for all the EVOOs with storage time. The increase and decrease in intensity of these peaks can be understood as the increase or decrease in the concentration of the compounds giving rise to the fluorescence signal. Typically, in the presence of light, the oil oxidation is accelerated in the presence of photosensitizers such as chlorophylls [34]. The EVOOs contain a considerable amount of chlorophyll to promote the photo-oxidation by acting as a sensitizer in the presence of light.

The increase in those peaks showing the local maxima of the standard deviation (Fig. 3), i.e., 420, 440, 464, and $515 \mathrm{~nm}$, was monitored with respect to the decrease of peak related to chlorophyll, i.e., $671 \mathrm{~nm}$. The variation of any particular peak was calculated with respect to the first day observations. Since the calculated difference in the peaks resulting from a decrease will result in a negative value, all values resulting from the decrease of $671 \mathrm{~nm}$ peaks were multiplied by -1 . The linear fit functions are presented in Fig. 4, 5, and 6. Furthermore, the extracted slope values for the three EVOO samples are presented in Fig. 7.

As illustrated in Fig. 7, for all the wavelengths, $420 \mathrm{~nm}$ (dark blue), $440 \mathrm{~nm}$ (sky blue), $464 \mathrm{~nm}$ (green), and $515 \mathrm{~nm}$ (yellow), Cornicabra oil sample showed the highest rate of evolution, followed by Arbequina and then Picual samples, when compared to the changes in peak at $671 \mathrm{~nm}$. The
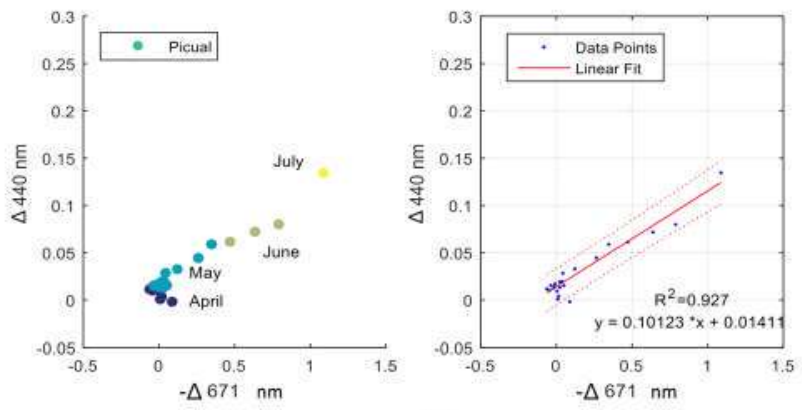

(b)
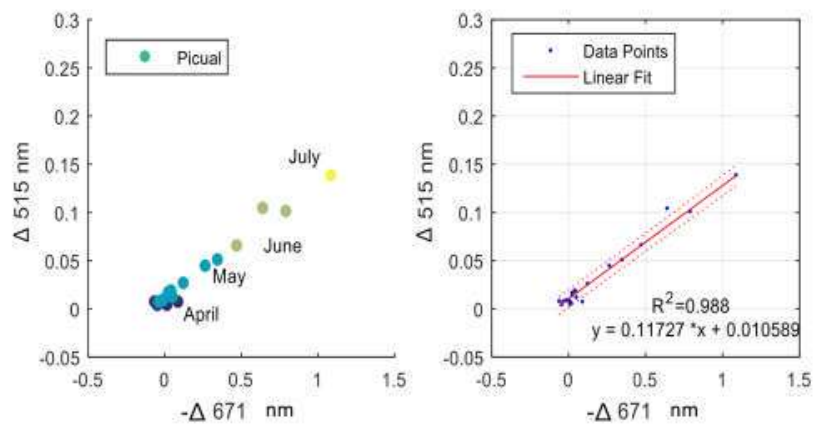

(d)

$515 \mathrm{~nm}$ (d) (y-axis). The oils were monitored for 4 months April, May, June, and July, which are marked in dark blue, sky blue, green, and yellow, respectively 

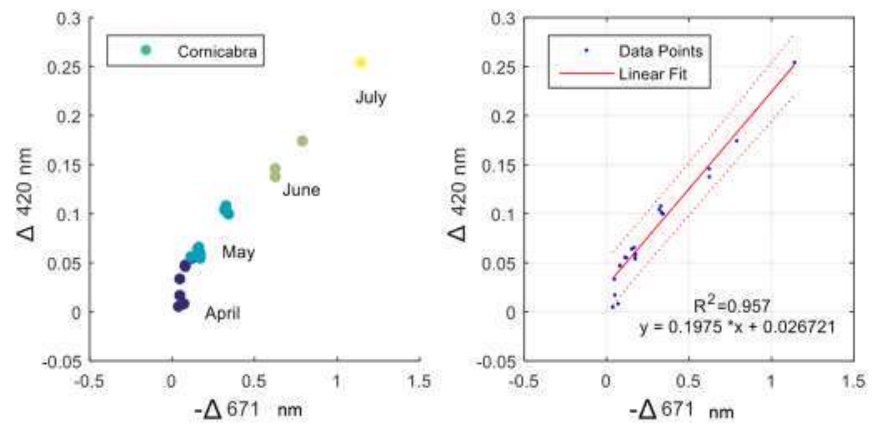

(a)
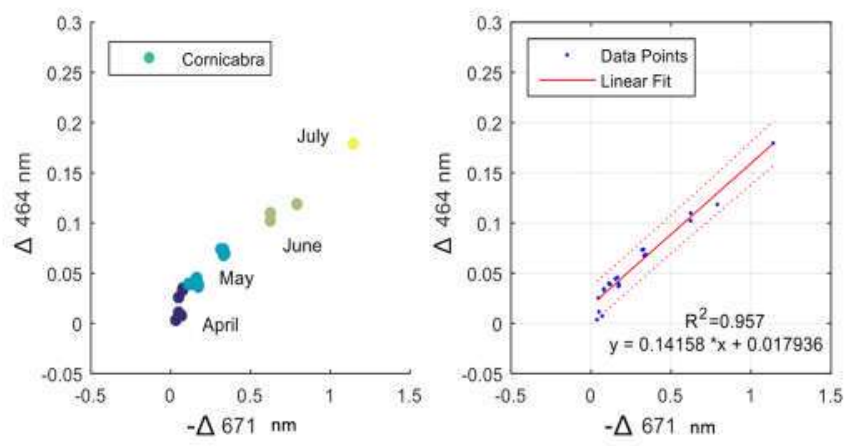

(c)

Fig. 6 Cornicabra oil evolution with storage time and the fitted linear curve with $95 \%$ confidence. The evolution is presented individually for $671 \mathrm{~nm}$ ( $x$-axis) vs $420 \mathrm{~nm}$ (a), $440 \mathrm{~nm}$ (b), $464 \mathrm{~nm}$ (c), and

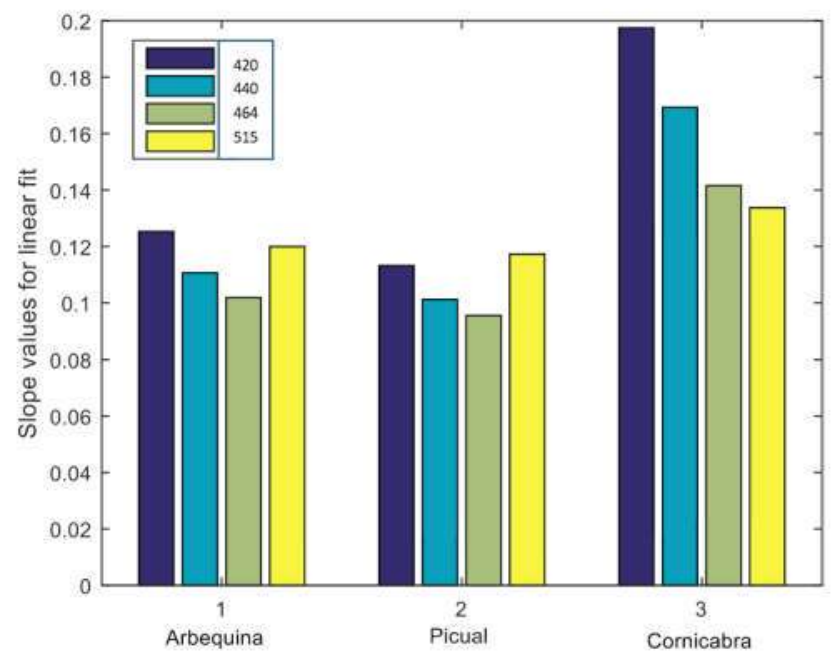

Fig. 7 Bar plot of the slopes obtained from the linear fit from data in Figs. 4, 5, and 6. The slope of the linear fit was understood as an estimate of the rate of evolution. The four colours show the four wavelengths used for monitoring (considering $420 \mathrm{~nm}$ in blue, $440 \mathrm{~nm}$ in sky blue, $464 \mathrm{~nm}$ in green, and $515 \mathrm{~nm}$ in yellow)

analysis of the slopes also revealed a different tendency at $510 \mathrm{~nm}$ for Cornicabra, showing the lowest value among the four wavelengths, whereas for Arbequina and Picual, the
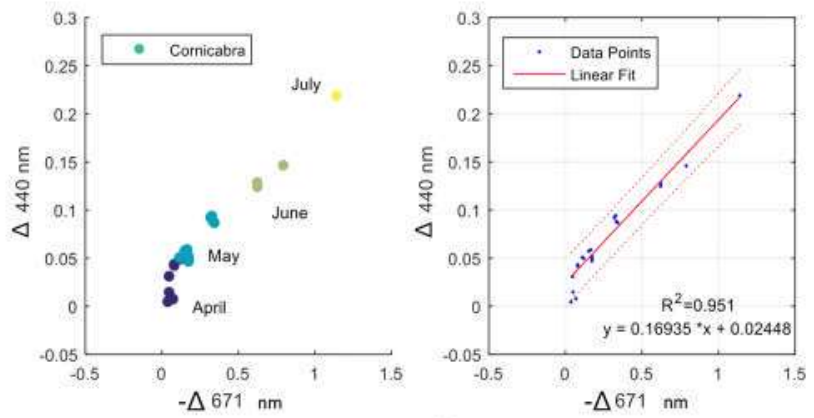

(b)
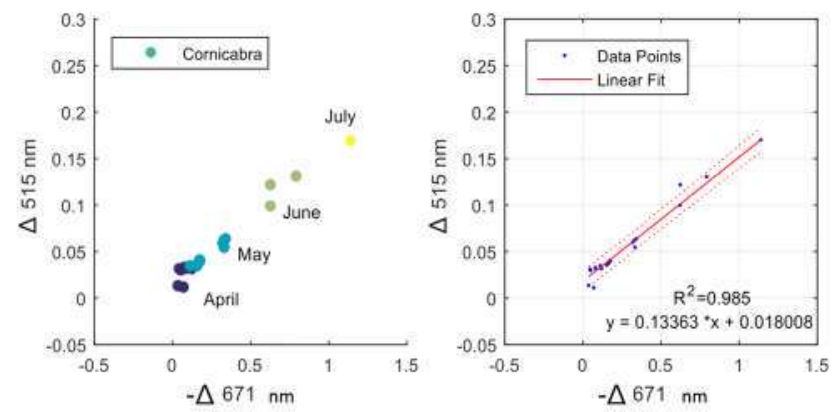

(d)

$515 \mathrm{~nm}$ (d) ( $y$-axis). The oils were monitored for 4 months April, May, June, and July, which are marked in dark blue, sky blue, green, and yellow, respectively

increase of this peak was noticeably higher than the other peaks. A reason for the different evolution rates obtained in Fig. 7 for different EVOO samples could be the different biochemical composition resulting from the presence of minor components such as free fatty acids, mono and diacylglycerols, metals, phospholipids, peroxides, chlorophylls, carotenoids, phenolic compounds, and tocopherols [34]. However, in the presented work, no chemical analysis was performed to validate that which particular underlying chemical compounds were causing the differences in the rate of evolution of EVOO samples. The obtained results could be included in a complete methodology for computation and comparison of different rates of deterioration in olive oil samples. Further research is needed with an increased number of samples and analytical reference techniques for computation of robust models.

\section{Comparison between exposure to light and darkness}

To study the evolution of a sample under different illumination conditions, two Picual samples (P2 and P3) were divided into two aliquots (P2_D, for dark; P2_L, for light; P3_D, for dark; P3_L, for light) and placed under dark and light conditions. These four samples were monitored for 6 months (from June to December 2015). The obtained 


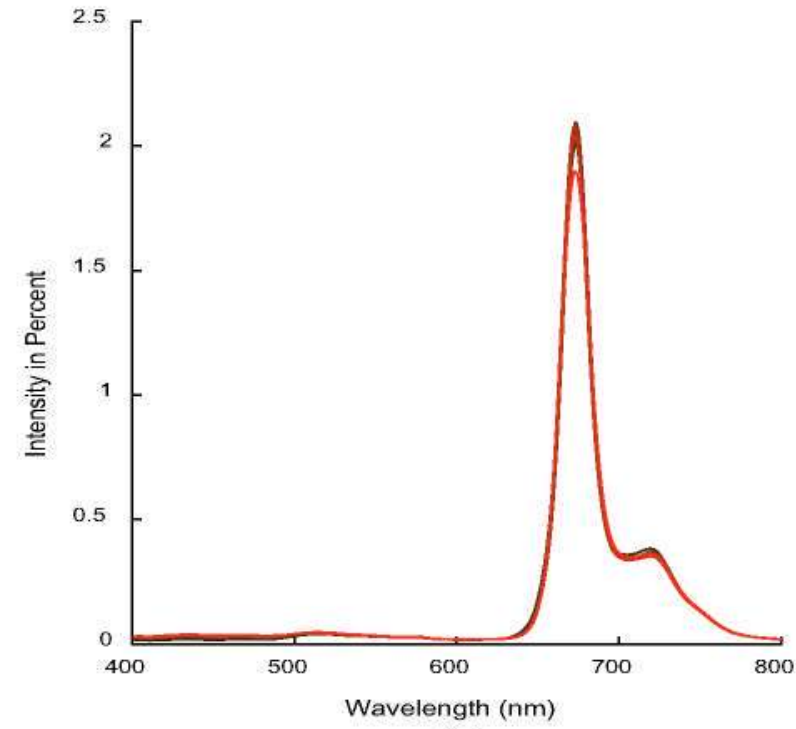

(a)

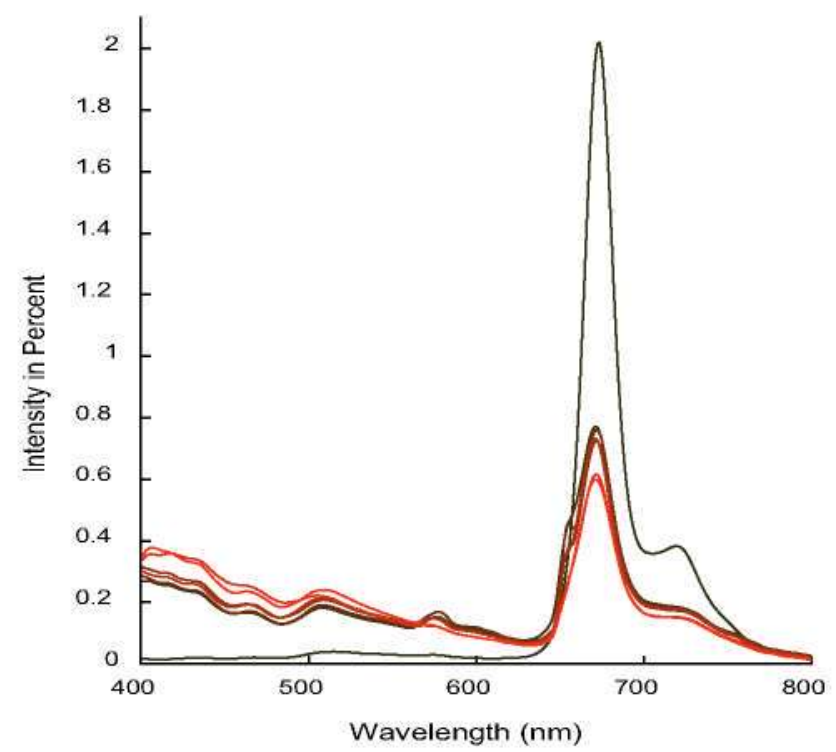

(c)

Fig. 8 Fluorescence spectra evolution obtained for the oils placed in the dark and exposed to light conditions. The spectra are presented after the pre-processing. a Picual (P2_D) sample placed in dark, b Picual (P3_D) sample placed in dark, $\mathbf{c}$ Picual (P2_L) sample placed

fluorescence spectra from the four samples are presented in Fig. 8. Figure $8 \mathrm{a}$, b shows the spectra evolution for the oil samples placed in dark conditions. Figure $8 \mathrm{c}$, d, shows the spectra evolutions of oil samples exposed to light conditions. In accordance with the results of the previous part of the experiment, the peaks within the region of 400-650 nm increased in intensity, whereas the peaks beyond $650 \mathrm{~nm}$ decreased with the storage time.

Figure 9 presents the total amount of variation (expressed in percentage) induced in the fluorescence spectra of oils

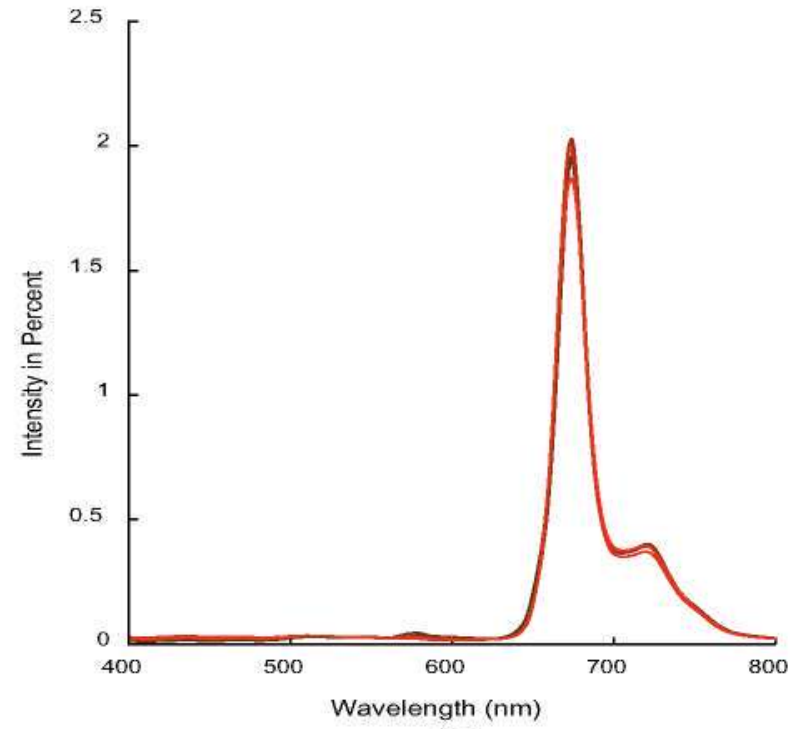

(b)

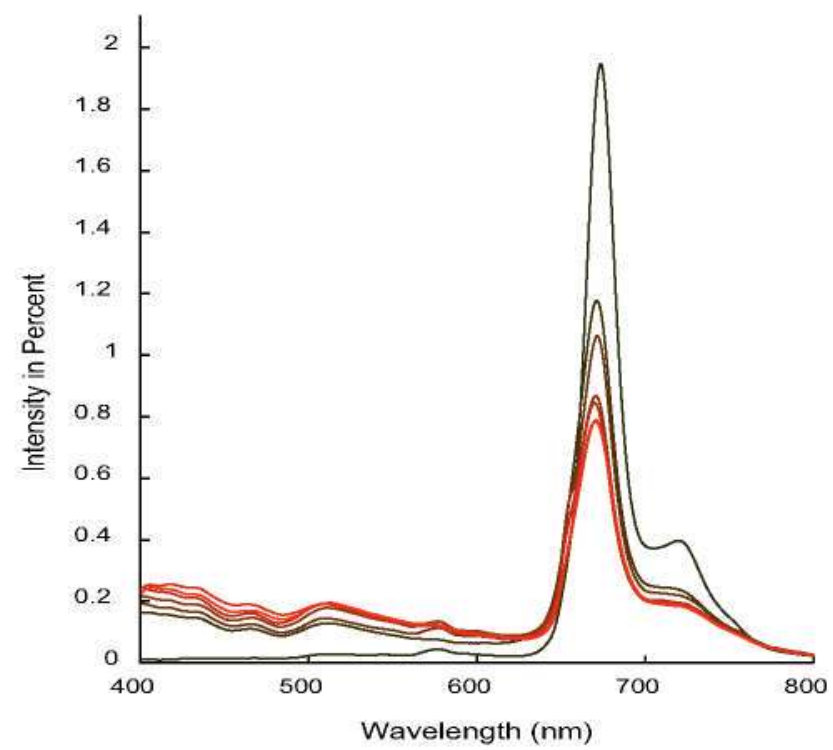

(d)

in the light, and $\mathbf{d}$ Picual $\mathrm{P}\left(3 \_\mathrm{L}\right)$ sample placed in light. The spectra in black represent the first recording, while the lightest red signifies the last recordings

after the 6 months of storage (M6) under different conditions. The percentage of variation was calculated with respect to the fluorescence spectra obtained on the first experimental date (M0). It can be easily noted from Fig. 8 that the spectra of oils exposed to light showed a huge percent of variation than the spectra of oils placed in the dark. It can also be seen that from 400 to $650 \mathrm{~nm}$, the variation is positive and after the $650 \mathrm{~nm}$ the variation is presented in negative, in accordance with the previous part of the study (Figs. 4, 5, 6). 


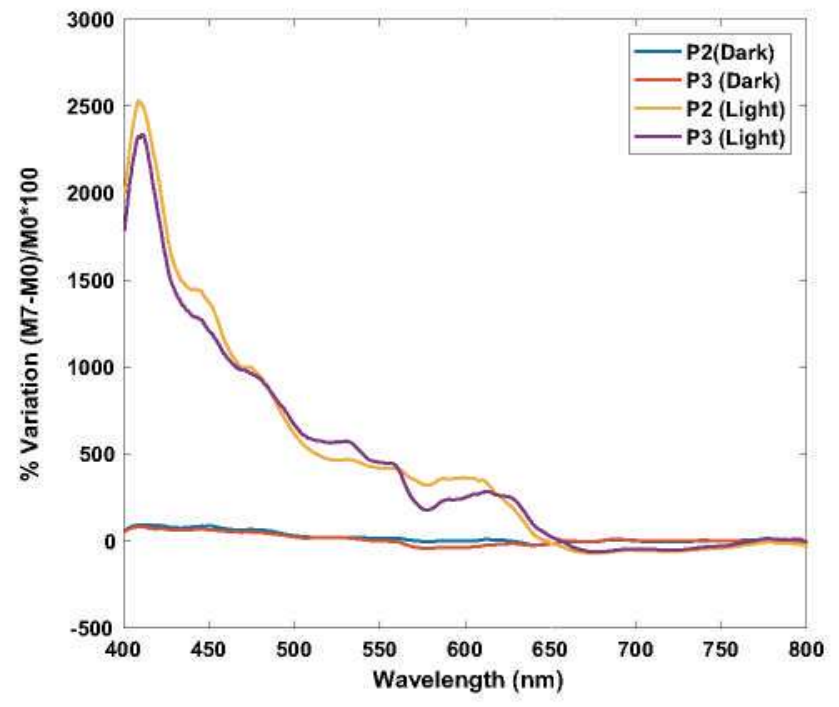

Fig. 9 Percentage of variation of the spectra of oil samples after the 6 months of storage (M6) in dark and exposed to light Picual Sample (P2 and P3). The percentage was calculated with respect to the spectra obtained on the first day of the experiment (M0)

Figure 9 also revealed that the oil aliquots kept in dark conditions showed a slight variation in the region of $400-515 \mathrm{~nm}$, while insignificant variation in the region above $650 \mathrm{~nm}$ dominated by chlorophyll was undergone. Such result can be seen as an evidence of the auto-oxidation process.

In the case of oil exposed to light, the pigment chlorophylls act as a photosensitizer to produce the singlet oxygen ${ }^{1} \mathrm{O}_{2}$, leading to an accelerated oxidation of EVOO. On the other hand, in the case of oil placed in the dark, the pigment chlorophylls act as antioxidants by donating hydrogen to free radicals [35]. The phenolic compounds decrease the autoxidation of oil in the dark through a variety of mechanisms based on radical scavenging, hydrogen atom transfer and metal-chelating [36]. In the present work, there was no chemical analysis performed to estimate the initial and final concentrations of any particular minor compound in the EVOO samples; therefore, no conclusions regarding the functioning of different minor compounds during oxidation can be made. Validation of the proposed methodology with a larger number of samples and chemical characterization is a major issue for the direction of our future works.

\section{Conclusion}

The oxidative stability of the extra virgin olive oils (EVOOs) is of high importance to prevent the formation of off-flavour compounds for maintaining its quality during storage. EVOOs can undergo auto-oxidation and photosensitized oxidation during the processing and storage which can result in loss of minor components responsible for sensory and nutritional quality. In the present study, a rapid nondestructive monitoring of oxidation changes in EVOOs was performed with the help of a right-angle prototype based on fluorescence spectroscopy. With simultaneous excitation in the range of $200-400 \mathrm{~nm}$ and recording fluorescence signatures in the range of $400-850 \mathrm{~nm}$, the prototype provided a fast monitoring of commercial EVOOs.

The major changes in the fluorescence spectra of EVOOs during the light exposure were associated with a decrease in fluorescence emission intensity at $671 \mathrm{~nm}$, and simultaneous increase at $420,440,464$, and $515 \mathrm{~nm}$, resulting due to a decrease in chlorophyll content and an increase in oxidation products, respectively. The evolution of the increasing peaks intensity $(420,440,464$, and $515 \mathrm{~nm})$ found to be linearly related to the decrease of the chlorophyll peak $(671 \mathrm{~nm})$ during oxidation processes. Monitoring of samples stored under dark conditions highlighted the auto-oxidation process, since no variation was found for fluorescence signal arising from chlorophyll, whereas the peaks related to oxidation products showed a little-increased variation. The fluorescence spectroscopy-based prototype could be proposed as a fast inexpensive screening tool in combination with high-resolution analytical techniques such as nuclear magnetic resonance (NMR) and high-performance liquid chromatography (HPLC), to develop classification protocols for EVOO samples.

Acknowledgements Authors gratefully acknowledge the Centro Tecnológico Agroalimentario de Lugo (Spain), Comunidad de Madrid (S2013/ABI-2747, TAVS-CM, Spain), and European Structural Funds for financial support. In addition, authors gratefully acknowledge Juan Ramón Izquierdo from the Laboratorio Arbitral Agroalimentario of MAGRAMA (Spain) for expert advice on olive oil quality. LPFTAGRALIA is a part of the CEI Moncloa Campus of Excellence, UPM-UCM (Spain).

Compliance with ethical standards

Conflict of interest The authors declare no conflict of interest.

Compliance with ethics requirements This article does not contain any studies with human and animal subjects.

\section{References}

1. Jaber H, Ayadi M, Makni J, Rigane G, Sayadi S, Bouaziz M (2012) Stabilization of refined olive oil by enrichment with chlorophyll pigments extracted from Chemlali olive leaves. Eur J Lipid Sci Technol 114(11):1274-1283

2. Sánchez-López E, Sánchez-Rodríguez MI, Marinas A, Marinas JM, Urbano FJ, Caridad JM, Moalem M (2016) Chemometric study of Andalusian extra virgin olive oils Raman spectra: qualitative and quantitative information. Talanta 156:180-190 
3. Karoui R, Blecker C (2011) Fluorescence spectroscopy measurement for quality assessment of food systems-a review. Food Bioprocess Technol 4(3):364-386

4. Kassouf A. El Rakwe M, Chebib H, Ducruet V, Rutledge DN, Maalouly J (2014) Independent components analysis coupled with $3 D$-front-face fuorescence spectroscopy to study the interaction between plastic food packaging and olive oil. Anal Chim Acta 839:14-25

5. Caponio F, Bilancia MT. Pasqualone A. Sikorska E, Gomes T (2005) Influence of the exposure to light on extra virgin olive oil quality during storage. Eur Food Res Technol 221(1-2):92-98

6. Velasco J, Dobarganes C (2002) Oxidative stability of virgin olive oil. Eur J Lipid Sci Technol 104(9-10):661-676

7. Frankel EN (2010) Chemistry of extra virgin olive oil: adulteration, oxidative stability. and antioxidants. J Agric Food Chem $58(10): 5991-6006$

8. Inarejos-García AM, Gómez-Alonso S, Fregapane G. Salvador MD (2013) Evaluation of minor components, sensory characteristics and quality of virgin olive oil by near infrared (NIR) spectroscopy. Food Res Int 50(1):250-258

9. Servili M. Esposto S. Taticchi A. Urbani S, Di Maio I, Veneziani G, Selvaggini R (2015) New approaches to virgin olive oil quality. technology, and by-products valorization. Eur J Lipid Sci Technol $117(11): 1882-1892$

10. Rahmani M, Csallany AS (1998) Role of minor constituents in the photooxidation of virgin olive oil. J Am Oil Chem Soc $75(7): 837-843$

11. Sikorska E. Khmelinskii IV. Sikorski M. Caponio F, Bilancia MT, Pasqualone A, Gomes T (2008) Fluorescence spectroscopy in monitoring of extra virgin olive oil during storage. Int J Food Sci Technol 43(1):52-61

12. Aparicio R. Roda L. Albi MA, Gutierrez F (1999) Effect of various compounds on virgin olive oil stability measured by Rancimat. J Agric Food Chem 47(10):4150-4155

13. Salvador MD, Aranda F, Fregapane G (1999) Contribution of chemical components of Cornicabra virgin olive oils to oxidative stability. A study of three successive crop seasons. J Am Oil Chem Soc 76(4):427-432

14. Gutierrez. F. Fernandez IL (2002) Determinant parameters and components in the storage of virgin olive oil. Prediction of storage time beyond which the oil is no longer of "extra" quality. J Agric Food Chem 50(3):571-577

15. Reboredo-Rodríguez P, González-Barreiro C. Cancho-Grande B, Simal-Gándara J (2012) Dynamic headspace/GC-MS to control the aroma fingerprint of extra-virgin olive oil from the same and different olive varieties. Food Control 25(2):684-695

16. Reboredo-Rodríguez P, Rey-Salgueiro L, Regueiro J, GonzálezBarreiro C, Cancho-Grande B, Simal-Gándara J (2014) Ultrasound-assisted emulsification-microextraction for the determination of phenolic compounds in olive oils. Food Chem 150:128-136

17. Reboredo-Rodríguez P, González-Barreiro C, Cancho-Grande B, Simal-Gándara J (2014) Quality of extra virgin olive oils produced in an emerging olive growing area in north-western Spain. Food Chem 164:418-426

18. Reboredo-Rodríguez. P. González-Barreiro C. Cancho-Grande B. Fregapane G. Salvador MD, Simal-Gándara J (2015) Characterisation of extra virgin olive oils from Galician autochthonous varieties and their co-crushings with Arbequina and Picual cv. Food Chem 176:493-503

19. Reboredo-Rodríguez P. González-Barreiro C. Cancho-Grande B. Valli E, Bendini A, Toschi TG. Simal-Gandara J (2016)
Characterization of virgin olive oils produced with autochthonous Galician varieties. Food Chem 212:162-171

20. Reboredo-Rodríguez P. Valli E. Bendini A, Di LG, Simal-Gándara J, Gallina TT (2016) A widely used spectrophotometric assay to quantify olive oil biophenols according to the health claim (EU Reg. 432/2012). Eur J Lipid Sci Technol 1 18(10):1593-1599

21. Guzmán E, Baeten V, Pierna JA, García-Mesa JA (2015) Evaluation of the overall quality of olive oil using fuorescence spectroscopy. Food Chem 173:927-934

22. Valli E, Bendini A, Berardinelli A, Ragni L, Riccò B, Grossi M, Gallina Toschi T (2016) Rapid and innovative instrumental approaches for quality and authenticity of olive oils. Eur J Lipid Sci Technol 118:1601-1619

23. Hernández-Sánchez N. Lleó L. Ammari F. Cuadrado TR, Roger JM (2017) Fast fluorescence spectroscopy methodology to monitor the evolution of extra virgin olive oils under illumination. Food Bioprocess Technol 10(5):949-961

24. Kyriakidis NB. Skarkalis P (2000) Fluorescence spectra measurement of olive oil and other vegetable oils. J AOAC Int 83(6): 1435-1439

25. Sikorska E, Górecki T, Khmelinskii IV, Sikorski M, Kozioł J (2005) Classification of edible oils using synchronous scanning fluorescence spectroscopy. Food Chem 89(2):217-225

26. Zandomeneghi M. Carbonaro L, Caffarata C (2005) Fluorescence of vegetable oils: olive oils. J Agric Food Chem 53(3):759-766

27. Giungato P, Aveni M, Rana R, Notarnicola L (2004) Changes induced by frying process in extra-virgin olive oil. Industrie Alimentari. Italy, pp 369-375

28. Guimet F, Ferré J. Boqué R, Rius FX (2004) Application of unfold principal component analysis and parallel factor analysis to the exploratory analysis of olive oils by means of excitation-emission matrix fluorescence spectroscopy. Anal Chim Acta 515(1):75-85

29. Kalua CM. Bedgood DR, Bishop AG, Prenzler PD (2006) Discrimination of storage conditions and freshness in virgin olive oil. J Agric Food Chem 54(19):7144-7151

30. Cheikhousman R. Zude M. Bouveresse DJ. Léger CL. Rutledge DN, Birlouez-Aragon I (2005) Fluorescence spectroscopy for monitoring deterioration of extra virgin olive oil during heating. Anal Bioanal Chem 382(6): 1438-1443

31. Tena N, García-González DL. Aparicio R (2009) Evaluation of virgin olive oil thermal deterioration by fluorescence spectroscopy. J Agric Food Chem 57(22): 10505-10511

32. Savitzky A, Golay MJ (1964) Smoothing and differentiation of data by simplified least squares procedures. Anal Chem 36(8): 1627-1639

33. Galeano Díaz T. Durán Merás I. Correa CA. Roldán B, Rodríguez Cáceres MI (2003) Simultaneous fluorometric determination of chlorophylls $a$ and $b$ and pheophytins $a$ and $b$ in olive oil by partial least-squares calibration. J Agric Food Chem 51(24):6934-6940

34. Choe E. Min DB (2006) Mechanisms and factors for edible oil oxidation. Compr Rev Food Sci Food Saf 5(4):169-186

35. Gutiérrez-Rosales F. Garrido-Fernández J, Gallardo-Guerreto L, Gandul-Rojas B. Minguez-Mosquera MI (1992) Action of chlorophylls on the stability of virgin olive oil. J Am Oil Chem Soc 69(9):866-871

36. Pouliarekou E. Badeka A, Tasioula-Margari M. Kontakos S, Longobardi F, Kontominas MG (2011) Characterization and classification of Western Greek olive oils according to cultivar and geographical origin based on volatile compounds. J Chromatogr A $1218(42): 7534-7542$ 